\title{
Influência da topografia de superfície nanométrica na estabilidade primária de mini-implantes dentários
}

\author{
Influence of the nanometric surface topography on the primary stability of \\ dental mini-implants

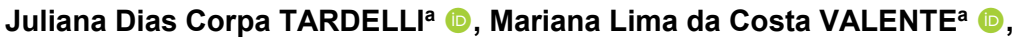 \\ Andréa Cândido DOS REIS ${ }^{a *}$ (i) \\ aUSP - Universidade de São Paulo, Faculdade de Odontologia de Ribeirão Preto, Ribeirão Preto, SP, Brasil
}

Como citar: Tardelli JDC, Valente MLC, dos Reis AC. Influência da topografia de superfície nanométrica na estabilidade primária de mini-implantes dentários. Rev Odontol UNESP. 2019;48:e20190019. https://doi.org/10.1590/1807-2577.01919

\begin{abstract}
Resumo
Introdução: A modificação físico-química da superfície de mini-implantes utilizados no suporte de overdentures pode influenciar o desempenho mecânico dos mesmos. Objetivo: Avaliar a influência de um tratamento de superfície do tipo nanométrico no desempenho mecânico de novos designs de miniimplantes. Material e método: Foram utilizados 40 mini-implantes (Ti-6Al-4V), com Ø $2 \mathrm{~mm} \times 10 \mathrm{~mm}$ de comprimento e dois designs diferentes, rosqueado e helicoidal, divididos em quatro grupos $(n=10)$, de acordo com o modelo e a presença ou a ausência de tratamento superficial. 0 desempenho mecânico foi avaliado por meio de torque de inserção e ensaio de arrancamento em cilindros ósseos suínos. Análise de variância ANOVA e teste de Tukey, com significância de 5\%, foram utilizados para análise estatística dos dados. Resultado: Foi observada diferença estatisticamente significante entre os grupos com e sem tratamento para torque de inserção $(p<0,001)$, e ensaio de arrancamento $(p=0,006)$, sendo a maior média para o grupo com tratamento, independentemente do design. Na comparação entre os designs, o rosqueado apresentou média significativamente maior $(\mathrm{p}<0,001)$ que o helicoidal. Conclusão: 0 tratamento de superfície nanométrico viabilizou melhor desempenho mecânico dos mini-implantes avaliados. Com relação aos novos designs testados, o rosqueado apresentou resultados superiores ao helicoidal.
\end{abstract}

Descritores: Implantes dentários; design; torque; alteração de superfície.

\begin{abstract}
Introduction: The physical-chemical modification of the surface of mini-implants used in the support of overdentures can influence the mechanical performance and survival of the same. Objective: To evaluate the influence of a nanometric surface treatment on the mechanical performance of new mini-implant designs. Material and method: 40 mini-implants (Ti-6Al-4V) with $\emptyset 2 \mathrm{~mm}$ x $10 \mathrm{~mm}$ in length and two different designs, threaded and helical, were divided into four groups $(\mathrm{n}=10)$ according to model, presence or absence of surface treatment. The mechanical performance was evaluated by means of insertion torque and pullout test in swine bony cylinders. Analysis of variance ANOVA and Tukey test, with significance of $5 \%$ were used for statistical analysis of the data. Result: A statistically significant difference was observed between the groups with and without treatment for insertion torque $(p<0.001)$ and pullout test $(p=0.006)$, being the highest mean for the treatment group, regardless of the design. In the comparison between the designs, the thread presented a significantly higher average $(p<0.001)$ than the helicoid. Conclusion: The nanometric surface treatment enabled better mechanical performance of the mini-implants evaluated. With respect to the new designs tested, the thread presented superior results to the helicoidal one.
\end{abstract}

Descriptors: Dental implants; design; torque; surface alteration. 


\section{INTRODUÇÃO}

Do ponto de vista da bioengenharia, dentre outras variáveis, a macrogeometria apresenta importante influência na área de contato osso/implante, na técnica cirúrgica, na ancoragem óssea e na distribuição de estresse na região peri-implantar ${ }^{1,2}$, fatores estes que influenciam diretamente a estabilidade primária no momento da instalação cirúrgica, responsável pelo feedback do tempo de cicatrização necessário para o carregamento da prótese e a sobrevivência do implante ${ }^{3}$.

Além da densidade, da quantidade óssea e do design do implante 4,5 , a estabilidade primária e, consequentemente, a osseointegração são influenciadas pelas modificações físico-químicas das superfícies, sendo a mais comum o aumento da rugosidade superficial, por meio de técnicas de condicionamento ácido, jateamento de areia ou oxidação anódica ${ }^{6}$. Embora este parâmetro seja um pré-requisito para a retenção de células osteogênicas e viabilize uma osteocondução mais rápida, pouco se discute sobre sua influência na estabilidade primária7 ${ }^{7}$.

A alteração na rugosidade dos implantes devido ao tratamento superficial possibilita maior coeficiente de atrito, contato osso/implante e torque de inserção, favorecendo a estabilidade inicial ${ }^{8-10}$.

As modificações superficiais podem ocorrer em escala macrométrica (mm), micrométrica $(\mu \mathrm{m})$ e nanométrica $(\mathrm{nm})^{11}$. Dentre os inúmeros métodos de texturização, a combinação de técnicas subtrativas e aditivas, como ataque ácido e tratamento alcalino, promove equilíbrio entre propriedades mecânicas e biológicas. 0 ácido cria micro e submicron porosidades na superfície, e o tratamento alcalino forma uma estrutura esponjosa com características submicron e nanométricas ${ }^{12}$.

Topografias nessas escalas melhoram a diferenciação dos osteoblastos, a proliferação celular e a estabilidade mecânica devido ao maior travamento do tecido ósseo adjacente ao implante. Tem sido relatado em vários estudos que a aplicação de nanoestruturas aumenta a bioatividade da superfície do implante e a aposição óssea ${ }^{13,14}$. Já segundo Rosa et al. ${ }^{15}$, as certezas são limitadas à influência do design do implante e da rugosidade da superfície apenas na escala micrométrica.

O desejo de reduzir o tempo de cicatrização óssea e proporcionar uma fase protética mais rápida ao paciente é responsável pelo desenvolvimento constante de novos modelos de implantes e tratamentos de superfície. Assim, o presente estudo avaliou a influência de um tratamento de superfície do tipo nanométrico na estabilidade primária de novos modelos de mini-implantes para overdentures, por meio de torque de inserção e ensaio de arrancamento, em cilindros ósseos suínos. A hipótese nula testada considerou que o tratamento de superfície não alteraria as propriedades mecânicas dos implantes avaliados.

\section{MATERIAL E MÉTODO}

\section{Implantes}

Foram utilizados 40 mini-implantes, confeccionados em liga de Ti-6Al-4V, com dimensões de $\emptyset 2 \mathrm{~mm} \times 10 \mathrm{~mm}$ de comprimento (Figura 1), divididos em quatro grupos ( $\mathrm{n}=10)$, de acordo com a Tabela 1 . 


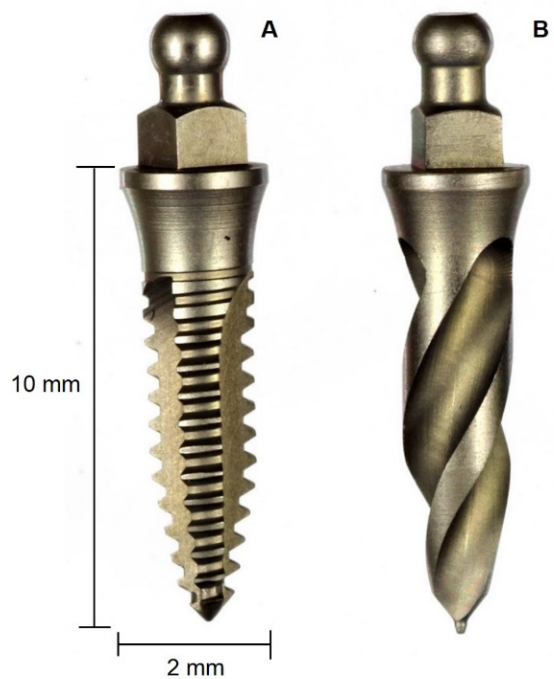

Figura 1. Mini-implantes: A. Modelo Rosqueado; B. Modelo Helicoidal.

Tabela 1. Características dos implantes selecionados

\begin{tabular}{cccc}
\hline Implante & Conexão protética & Formato & $\begin{array}{c}\text { Tratamento de } \\
\text { Superfície }\end{array}$ \\
\hline $\begin{array}{c}\text { Rosqueado } \\
\text { G1 }\end{array}$ & Corpo único & Corpo com roscas e três chanfros longitudinais e equidistantes & $\mathrm{H}_{3} \mathrm{PO}_{4}+\mathrm{NaOH}$ \\
$\begin{array}{c}\text { Rosqueado } \\
\text { G2 }\end{array}$ & Corpo único & Corpo com roscas e três chanfros longitudinais e equidistantes & Usinado \\
$\begin{array}{c}\text { Helicoidal } \\
\text { G3 }\end{array}$ & Corpo único & Corpo com apenas duas roscas de passo longo com \\
configuração helicoidal & $\mathrm{H}_{3} \mathrm{PO}_{4}+\mathrm{NaOH}$ \\
Helicoidal & Corpo único & Corpo com apenas duas roscas de passo longo com & \\
G4 & configuração helicoidal & Usinado \\
\hline
\end{tabular}

0 tratamento nanométrico de superfície dos mini-implantes consistiu em ataque ácido $\left(\mathrm{H}_{3} \mathrm{PO}_{4}\right)$ seguido de tratamento alcalino com hidróxido de sódio $(\mathrm{NaOH})^{16}$.

\section{Protocolo de Inserção}

Os mini-implantes foram inseridos individualmente em cilindros ósseos suínos, com dimensões de $\emptyset 10 \mathrm{~mm} \times 20 \mathrm{~mm}$ de comprimento, seguindo-se o protocolo de fresas indicado para cada design, com auxílio de um Motor Elétrico Cirúrgico MC 101 (Dentscler ${ }^{\circledR}$, Ribeirão Preto, São Paulo, Brasil), ajustado a um torque de 45 N e $1.350 \mathrm{rpm}$. 0 cilindro ósseo removido da cabeça do fêmur suíno foi utilizado devido às suas características semelhantes aos ossos maxilar e mandibular humanos na análise da estabilidade primária ${ }^{17}$.

\section{Análise das Propriedades Mecânicas}

Para análise das propriedades mecânicas, foram utilizados o torque de inserção (TI) e ensaio de arrancamento (ASTM F543), método empregado em análises laboratoriais ${ }^{5,18,19}$, capaz de avaliar a resistência dos implantes (dentais ou ortopédicos; metálicos ou não) ao deslocamento vertical (força máxima), em associação às características do design e do material de inserção.

O TI máximo dos mini-implantes foi aferido por meio de um torquímetro até o completo assentamento dos mesmos no tecido ósseo.

0 ensaio de arrancamento foi realizado em uma Máquina Universal de Ensaios Emic DL10000 (São José dos Pinhais, São Paulo, Brasil). Através de um dispositivo confeccionado 
especificamente para o estudo, os mini-implantes foram fixados no equipamento e submetidos a uma força axial de tração com velocidade constante de $2 \mathrm{~mm} / \mathrm{min}$ e célula de carga de $200 \mathrm{Kg}$. Em todos os mini-implantes, foi utilizada pré-carga de $10 \mathrm{~N}$ e tempo de acomodação de 30 segundos, valores previamente definidos. Um software Tesc acoplado à máquina forneceu os valores de força máxima de arrancamento em newtons $(\mathrm{N})$.

\section{Análise Estatística}

Os dados foram avaliados com o programa SPSS 20.0 Statistical Software (Statistical Package for the Social Sciences, Nova York, EUA), por meio de análise de variância (ANOVA) e teste de Tukey $(\alpha=0,05)$.

\section{RESULTADO}

\section{Torque de Inserção}

Não houve interação entre os fatores implante e tratamento de superfície $(p=0,096)$. Considerando os dois modelos de mini-implantes, o rosqueado apresentou média significativamente maior $(\mathrm{p}<0,001)$ que o helicoidal. Os mini-implantes com tratamento de superfície demostraram maior média $(\mathrm{p}<0,001)$ quando comparados aos sem tratamento (Tabela 2).

Tabela 2. Média e desvio padrão do torque de inserção (N.cm) dos mini-implantes com e sem tratamento de superfície

\begin{tabular}{cccc}
\hline \multirow{2}{*}{ Implante } & \multicolumn{3}{c}{ Tratamento de Superfície } \\
\cline { 2 - 4 } & Com & Sem & Média \\
\hline Rosqueado & $29,60(5,37)$ & $23,10(7,10)$ & $26,35(6,98)^{\mathrm{a}}$ \\
Helicoidal & $23,70(6,07)$ & $11,30(1,63)$ & $17,50(7,69)^{\mathrm{b}}$ \\
Média & $26,65(6,352)^{\mathrm{a}}$ & $17,20(7,86)^{\mathrm{b}}$ & \\
\hline
\end{tabular}

Letras minúsculas diferentes na mesma coluna indicam diferença estatística.

\section{Ensaio de Arrancamento}

Não houve interação entre os fatores implante e tratamento de superfície $(p=0,609)$. Considerando os designs dos modelos de mini-implantes, o rosqueado apresentou média significativamente maior $(\mathrm{p}<0,001)$ que o helicoidal. Com relação ao tratamento superficial, os mini-implantes com tratamento demostraram maior média $(\mathrm{p}=0,006)$ quando comparados aos sem tratamento (Tabela 3).

Tabela 3. Média e desvio padrão do ensaio de arrancamento $(\mathrm{N})$ dos mini-implantes com e sem tratamento de superfície

\begin{tabular}{cccc}
\hline \multirow{2}{*}{ Implante } & \multicolumn{3}{c}{ Tratamento de Superfície } \\
\cline { 2 - 4 } & Com & Sem & Média \\
\hline Rosqueado & $218,69(26,65)$ & $194,46(52,32)$ & $206,58(42,28)^{\mathrm{a}}$ \\
Helicoidal & $78,79(22,97)$ & $44,16(9,11)$ & $61,47(24,59)^{\mathrm{b}}$ \\
Média & $148,74(75,74)^{\mathrm{a}}$ & $119,31(85,33)^{\mathrm{b}}$ & \\
\hline
\end{tabular}

Letras minúsculas diferentes na mesma coluna indicam diferença estatística. 


\section{DISCUSSÃO}

A estabilidade primária é um dos aspectos clínicos mais importantes no tratamento com implantes e é influenciada por fatores, como design, características da topografia de superfície, qualidade óssea e técnica cirúrgica ${ }^{20}$. Os dados do presente estudo rejeitam a hipótese nula, pois confirmaram a ação positiva do tratamento nanométrico de superfície no desempenho mecânico dos mini-implantes, quando comparados aos respectivos modelos usinados.

Os mini-implantes rosqueado e helicoidal, independentemente do design, apresentaram melhor embricamento mecânico, demonstrado pelo torque de inserção e ensaio de arrancamento, quando comparados aos respectivos modelos usinados. Isso se deve provavelmente à maior rugosidade da superfície, criada pelo tratamento nanométrico, como demonstrado anteriormente ${ }^{6,21}$.

Estes resultados corroboram com os de Mazzo et al. ${ }^{8}$, que verificaram maior resistência ao arrancamento de implantes com superfície tratada por ataque ácido. De acordo com os autores, esses achados sugerem que a rugosidade causada pelo tratamento superficial aumenta a fricção entre a superfície implante/osso, influenciando a estabilidade primária. Wennerberg et al. ${ }^{22}$ também observaram maior torque de remoção e contato ósseo interfacial em implantes de titânio com superfície tratada por jateamento em detrimento das superfícies usinadas.

Contrariamente, Gansukh et al. ${ }^{23}$ verificaram um torque de inserção menor em miniimplantes ortodônticos com superfície tratada, quando comparada à usinada, e, de acordo com eles, isso é devido à redução do diâmetro do implante pelo processo de texturização ${ }^{24}$. Esses resultados vão de encontro aos observados neste estudo, em que, embora os implantes avaliados também apresentassem diâmetro reduzido, o tratamento nanométrico aplicado promoveu maior torque de inserção.

0 tratamento de superfície melhorou a estabilidade primária dos mini-implantes independentemente do design; no entanto, os modelos apresentaram diferenças estatísticas quando comparados entre si quanto ao torque de inserção e no ensaio de arrancamento. Neste caso, o design rosqueado proporcionou melhores resultados que o helicoidal, com valor médio de torque de inserção mais próximo ao ideal para o carregamento imediato ${ }^{25}$.

A estabilidade primária também sofre influência direta da área de contato osso/implante, principalmente devido às características associadas ao design (forma, largura, profundidade, inclinação, face e ângulo da hélice) e ao passo de rosca (ângulo e largura). Baseado nisso, o intertravamento mecânico do modelo rosqueado foi superior ao helicoidal, provavelmente devido ao menor passo de rosca, o qual, além de aumentar a área de superfície, melhora a compactação óssea e, consequentemente, o torque de inserção ${ }^{26,27}$. Já no helicoidal, a macrogeometria com apenas duas roscas de passo longo pode ter gerado menor fricção implante/osso, reduzindo a estabilidade inicial no modelo ósseo avaliado ${ }^{8,28}$.

Falco et al. ${ }^{29}$, por outro lado, observaram torque de inserção superior $(37,96 \pm 11,78)$ e menor micromovimentação em implantes com maior passo de rosca, quando avaliados em ossos de baixa densidade. Diferentemente do observado no presente estudo, no qual o formato do implante helicoidal - com maior passo de rosca - associado ao osso suíno de maior densidade promoveu menor torque de inserção.

O sucesso de uma reabilitação com implantes é influenciado, entre outros fatores, pelo torque de inserção e pelo tratamento de superfície, responsáveis pelo travamento mecânico, remodelação e aposição ${ }^{30,31}$. Dessa forma, a análise desses parâmetros pode fornecer um conhecimento mais profundo sobre o desempenho mecânico de novos modelos de implantes. 


\section{CONCLUSÃO}

A realização do tratamento de superfície do tipo nanométrico sugere melhor desempenho mecânico dos mini-implantes, independentemente do design. Na comparação entre os modelos, o rosqueado apresentou melhores resultados em relação ao helicoidal.

\section{AGRADECIMENTOS}

Os autores agradecem ao Conselho Nacional de Desenvolvimento Científico e Tecnológico (CNPq) pelo suporte financeiro à presente pesquisa [164516/2017-4]

\section{REFERÊNCIAS}

1. Toyoshima T, Wagner W, Klein MO, Stender E, Wieland M, Al-Nawas B. Primary stability of a hybrid self-tapping implant compared to a cylindrical non-self-tapping implant with respect to drilling protocols in an ex vivo model. Clin Implant Dent Relat Res. 2011 Mar;13(1):71-8. http://dx.doi.org/10.1111/j.1708-8208.2009.00185.x. PMid:19681929.

2. Sciasci P, Casalle N, Vaz LG. Evaluation of primary stability in modified implants: Analysis by resonance frequency and insertion torque. Clin Implant Dent Relat Res. 2018 Jun;20(3):274-9. http://dx.doi.org/10.1111/cid.12574. PMid:29380490.

3. Walker LR, Morris GA, Novotny PJ. Implant insertional torque values predict outcomes. J Oral Maxillofac Surg. 2011 May;69(5):1344-9. http://dx.doi.org/10.1016/j.joms.2010.11.008. PMid:21398013.

4. Iezzi G, Scarano A, Di Stefano DA, Arosio P, Doi K, Ricci L, et al. Correlation between the bone density recorded by a computerized implant motor and by a histomorphometric analysis: a preliminary in vitro study on bovine ribs. Clin Implant Dent Relat Res. 2015 Jan;17(Suppl 1):e35-44. http://dx.doi.org/10.1111/cid.12121. PMid:23879771.

5. Valente ML, de Castro DT, Shimano AC, Lepri CP, dos Reis AC. Analyzing the Influence of a New Dental Implant Design on Primary Stability. Clin Implant Dent Relat Res. 2016 Feb;18(1):168-73. http://dx.doi.org/10.1111/cid.12324. PMid:25801693.

6. Oue H, Doi K, Oki Y, Makihara Y, Kubo T, Perrotti V, et al. Influence of implant surface topography on primary stability in a standardized osteoporosis rabbit model study. J Funct Biomater. 2015 Mar;6(1):143-52. http://dx.doi.org/10.3390/jfb6010143. PMid:25794350.

7. Elias CN, Rocha FA, Nascimento AL, Coelho PG. Influence of implant shape, surface morphology, surgical technique and bone quality on the primary stability of dental implants. J Mech Behav Biomed Mater. 2012 Dec;16:169-80. http://dx.doi.org/10.1016/j.jmbbm.2012.10.010. PMid:23182386.

8. Mazzo CR, Reis AC, Shimano AC, Valente ML. In vitro analysis of the influence of surface treatment of dental implants on primary stability. Braz Oral Res. 2012 Jul-Aug;26(4):313-7. http://dx.doi.org/10.1590/S1806-83242012005000006. PMid:22596223.

9. Fröjd V, Wennerberg A, Franke Stenport V. Importance of Ca2+ modifications for osseointegration of smooth and moderately rough anodized titanium implants - a removal torque and histological evaluation in rabbit. Clin Implant Dent Relat Res. 2012 Oct;14(5):737-45. http://dx.doi.org/10.1111/j.1708-8208.2010.00315.x. PMid:20977616.

10. Carmo Filho LCD, Marcello-Machado RM, Castilhos ED, Del Bel Cury AA, Faot F. Can implant surfaces affect implant stability during osseointegration? A randomized clinical trial. Braz Oral Res. 2018 Oct;32(0):e110. http://dx.doi.org/10.1590/1807-3107bor-2018.vol32.0110. PMid:30379212.

11. Svanborg LM, Andersson M, Wennerberg A. Surface characterization of commercial oral implants on the nanometer level. J Biomed Mater Res B Appl Biomater. 2010 Feb;92(2):462-9. PMid:19957360. 
12. Claros CAE, Oliveira DP, Campanelli LC, Pereira da Silva PSC, Bolfarini C. Fatigue behavior of Ti-6Al-4V alloy in saline solution with the surface modified at a micro- and nanoscale by chemical treatment. Mater Sci Eng C Mater Biol Appl. 2016 Oct;67:425-32. http://dx.doi.org/10.1016/j.msec.2016.04.099. PMid:27287139.

13. Bjursten LM, Rasmusson L, Oh S, Smith GC, Brammer KS, Jin S. Titanium dioxide nanotubes enhance bone bonding in vivo. J Biomed Mater Res A. 2010 Mar;92(3):1218-24. PMid:19343780.

14. Jimbo R, Sotres J, Johansson C, Breding K, Currie F, Wennerberg A. The biological response to three different nanostructures applied on smooth implant surfaces. Clin Oral Implants Res. 2012 Jun;23(6):706-12. http://dx.doi.org/10.1111/j.1600-0501.2011.02182.x. PMid:21488968.

15. Rosa MB, Albrektsson T, Francischone CE, Schwartz Filho HO, Wennerberg A. The influence of surface treatment on the implant roughness pattern. J Appl Oral Sci. 2012 Sep-Oct;20(5):550-5. http://dx.doi.org/10.1590/S1678-77572012000500010. PMid:23138742.

16. Oliveira DP, Prokofiev E, Sanches LFR, Polyakova V, Valiev RZ, Botta WJ, et al. Surface chemical treatment of ultrafine-grained Ti-6Al-7 Nb alloy processed by severe plastic deformation. J Alloys Compd. 2015 Sep;643(Suppl 1):S241-5. http://dx.doi.org/10.1016/j.jallcom.2014.11.115.

17. Pithon MM, Nojima MG, Nojima LI. Primary stability of orthodontic mini-implants inserted into maxilla and mandible of swine. Oral Surg Oral Med Oral Pathol Oral Radiol. 2012 Jun;113(6):748-54. http://dx.doi.org/10.1016/j.tripleo.2011.06.021. PMid:22677021.

18. Rittel D, Dorogoy A, Shemtov-Yona K. Modeling the effect of osseointegration on dental implant pullout and torque removal tests. Clin Implant Dent Relat Res. 2018 Oct;20(5):683-91. http://dx.doi.org/10.1111/cid.12645. PMid:30051951.

19. Valente MLDC, Castro DT, Shimano AC, Reis ACD. Influence of an alternative implant design and surgical protocol on primary stability. Braz Dent J. 2019 Jan-Feb;30(1):47-51. http://dx.doi.org/10.1590/0103-6440201902324. PMid:30864647.

20. Coelho PG, Jimbo R. Osseointegration of metallic devices: current trends based on implant hardware design. Arch Biochem Biophys. 2014 Nov;561:99-108. http://dx.doi.org/10.1016/j.abb.2014.06.033. PMid:25010447.

21. Elias CN, Meirelles L. Improving osseointegration of dental implants. Expert Rev Med Devices. 2010 Mar;7(2):241-56. http://dx.doi.org/10.1586/erd.09.74. PMid:20214429.

22. Wennerberg A, Albrektsson T, Andersson B. An animal study of $\mathrm{cp}$ titanium screws with different surface topographies. J Mater Sci Mater Med. 1995 May;6(5):302-9. http://dx.doi.org/10.1007/BF00120275.

23. Gansukh O, Jeong JW, Kim JW, Lee JH, Kim TW. Mechanical and histological effects of resorbable blasting media surface treatment on the initial stability of orthodontic mini-implants. BioMed Res Int. 2016;2016:7520959. http://dx.doi.org/10.1155/2016/7520959. PMid:26942200.

24. Maiorana C, Farronato D, Pieroni S, Cicciu M, Andreoni D, Santoro F. A four-year survival rate multicenter prospective clinical study on 377 implants: correlations between implant insertion torque, diameter, and bone quality. J Oral Implantol. 2015 Jun;41(3):e60-5. http://dx.doi.org/10.1563/AAIDJOI-D-13-00206. PMid:24517193.

25. Toth A, Hasan I, Bourauel C, Mundt T, Biffar R, Heinemann F. The influence of implant body and thread design of mini dental implants on the loading of surrounding bone: a finite element analysis. Biomed Tech (Berl). 2017 Aug;62(4):393-405. http://dx.doi.org/10.1515/bmt-2016-0002. PMid:28358711.

26. Griggs JA. Dental implants. Dent Clin North Am. 2017 Oct;61(4):857-71. http://dx.doi.org/10.1016/j.cden.2017.06.007. PMid:28886772.

27. Ma P, Xiong W, Tan B, Geng W, Liu J, Li W, et al. Influence of thread pitch, helix angle, and compactness on micromotion of immediately loaded implants in three types of bone quality: a three-dimensional 
finite element analysis. BioMed Res Int. 2014;2014:983103. http://dx.doi.org/10.1155/2014/983103. PMid:25110716.

28. Menezes HHM, Naves MM, Costa HL, Barbosa TP, Ferreira JA, Magalhães D, et al. Effect of surgical installation of dental implants on surface topography and its influence on osteoblast proliferation. Int J Dent. 2018 Oct;2018:4089274. http://dx.doi.org/10.1155/2018/4089274. PMid:30416524.

29. Falco A, Berardini M, Trisi P. Correlation between implant geometry, implant surface, insertion torque, and primary stability: in vitro biomechanical analysis. Int J Oral Maxillofac Implants. 2018 Jul/Aug;33(4):824-30. http://dx.doi.org/10.11607/jomi.6285. PMid:30024998.

30. Geckili O, Bilhan H, Geckili E, Cilingir A, Mumcu E, Bural C. Evaluation of possible prognostic factors for the success, survival, and failure of dental implants. Implant Dent. 2014 Feb;23(1):44-50. http://dx.doi.org/10.1097/ID.0b013e3182a5d430. PMid:24113554.

31. Yamaguchi Y, Shiota M, Munakata M, Kasugai S, Ozeki M. Effect of implant design on primary stability using torque-time curves in artificial bone. Int J Implant Dent. 2015 Dec;1(1):21. http://dx.doi.org/10.1186/s40729-015-0024-0. PMid:27747643.

\section{CONFLITOS DE INTERESSE}

Os autores declaram não haver conflitos de interesse.

\section{*AUTOR PARA CORRESPONDÊNCIA}

Andréa Cândido dos Reis, USP - Universidade de São Paulo, FORP - Faculdade de Odontologia de Ribeirão Preto, Avevida do Café, s/n, 14040-904 Ribeirão Preto - SP, Brasil, e-mail: andreare73@yahoo.com.br

Recebido: Março 8, 2019

Aprovado: Julho 11, 2019 